\title{
Valoración de los profesionales sobre la enseñanza del periodismo en España. Un análisis intergeneracional
}

\author{
Bernardo J. Gómez CALDERÓN \\ Universidad de Málaga \\ bjgomez@uma.es \\ Sergio Roses CAMPos \\ Universidad de Málaga \\ sergioroses@uma.es
}

Recibido: 24/04/2012

Aceptado: 19/11/2012

\section{Resumen}

Hace cuatro décadas que se implantaron los estudios universitarios de Periodismo en España. Durante este tiempo, la estructura de las enseñanzas ha estado sometida a numerosos cambios, orientados a adaptar la formación de los estudiantes a las exigencias del mercado de trabajo. En esta comunicación, los autores analizan la evolución de los estudios de Periodismo en nuestro país y la valoración que les merece a los profesionales la formación recibida en las facultades, con la hipótesis de que debe variar a lo largo del tiempo por los cambios acometidos en los planes de estudios. Los resultados apuntan a que no hay diferencias significativas en la opinión manifestada por periodistas de distintas generaciones, pero sí con respecto a los aspectos positivos y negativos destacados, confirmando el efecto beneficioso del incremento de materias prácticas en el currículo de la licenciatura.

Palabras clave: Universidad, Licenciatura de Periodismo, EEES, planes de estudios, profesión periodística.

\section{Professionals' Opinion about the Journalism Studies in Spain an Intergenerational Analysis}

\begin{abstract}
It is four decades since Journalism studies first began at Spanish universities in 1971. Since then, they have been subject to changes in their contents and methods to adjust to the social demands and students needs. In this paper, authors analyze the evolution of the Journalism studies in Spain, as well as the graduates' opinion about the education received. We wanted to know if there is a significant difference between the opinions that several generations express on the usefulness of the University studies, because of the evolution of the syllabuses. The results make clear that there are no differences in the general perception of the Journalism studies depending on the professionals' age. Nevertheless, we have found differences about the positive and negative aspects that every group emphasizes, and this confirms that the increase of practical matters in the syllabuses has been beneficial.

Keywords: University, Journalism studies, EHEA, syllabuses, journalistic profession.

\section{Referencia normalizada}

GÓMEZ CALDERÓN, Bernardo J. y ROSES CAMPOS, Sergio (2013): "Valoración de los profesionales sobre la enseñanza del periodismo en España. Un análisis intergeneracional". Estudios sobre el mensaje periodístico. Vol. 19, Núm. 1, págs.: 403-418. Madrid, Servicio de Publicaciones de la Universidad Complutense.
\end{abstract}

Sumario: 1. Introducción; 1.1. Configuración histórica de los estudios de Periodismo en España; 1.2. El debate en torno a la orientación de los estudios; 1.3. La adaptación al EEES; 1.4. Aproximaciones aca- 
démicas a la opinión de los profesionales sobre los estudios de Periodismo. 2. Objetivos e hipótesis. 3. Metodología; 3.1. Población, muestra y recolección de los datos; 3.2. Variables del estudio; 3.3. Procedimiento de análisis. 4. Resultados; 4. 1. Valoración de la formación recibida según la generación del periodista; 4. 2. Opinión sobre aspectos positivos y negativos según la generación del periodista; 4. 3 . Valoración de la formación recibida según el tipo de medio en el que trabaja. 5. Conclusiones y discusión. 6. Referencias bibliográficas.

\section{Introducción}

La progresiva extensión de los estudios universitarios de Periodismo en España, que en poco más de cuarenta años han pasado a engrosar la oferta de titulaciones de la mayor parte de universidades, tanto públicas como privadas, y que disfrutan de una demanda creciente por parte del alumnado, ha favorecido la reflexión sobre su estructura, sus fines y su efectividad de cara a la formación de los futuros profesionales de la información.

Las razones que explican su crecimiento "van desde un potente efecto de espejismo mediático en los jóvenes a una respuesta racional a la fuerte expansión económica de las industrias culturales" (Pestano et al., 2011: 408), y sus consecuencias no son siempre positivas, en especial debido a la saturación del mercado de trabajo, que, en plena crisis económica mundial, tiende a agudizarse.

Aunque, en términos cuantitativos, el peso de la titulación de Periodismo en el área de Ciencias Sociales y Jurídicas no deja de incrementarse, su contribución a las aptitudes y conocimientos que los periodistas egresados deben desarrollar y adquirir están sometidas a un escrutinio académico y profesional cada vez más intenso: se duda (se ha dudado siempre) de su contribución a la formación de periodistas bien preparados, y esta es una rémora difícil de erradicar.

La propia evolución del sistema de medios, en continua transformación, representa un reto constante para unos estudios condenados a moverse siempre entre el afianzamiento de sus bases teóricas y las exigencias del oficio. Los cambios producidos en el sector de la comunicación en los últimos veinte años han modificado los perfiles profesionales, y el nacimiento de nuevos medios, con Internet como soporte paradigmático, ha obligado a experimentar con lenguajes y formatos diferentes (López, 2009: web), lo cual ha llevado a reformular los planes de estudios coincidiendo con la implantación del Espacio Europeo de Educación Superior (EEES).

Dado que el proceso de Bolonia aún se está implementando en España, es imposible evaluar la eficacia de los nuevos grados. Pero con respecto a las licenciaturas que ahora se extinguen, sí que resulta posible indagar, y consideramos que un buen baremo para determinar su adecuación a las necesidades del ejercicio periodístico es la opinión de los propios profesionales. De ello nos ocupamos en este trabajo.

\subsection{Configuración histórica de los estudios de Periodismo en España}

En 1941 se creó la primera Escuela Oficial de Periodismo, en Madrid, dependiente de la Dirección General de Prensa, un organismo del Estado sujeto a los principios de propaganda del régimen franquista, pero que sirvió para que varias generaciones de periodistas accedieran a la profesión formadas siquiera esquemáticamente en los rudimentos de la comunicación. Entre los años 50 y 60, la Escuela Oficial de Perio- 
dismo extendió sus estudios a otras dos ciudades, Barcelona y La Laguna ${ }^{1}$. En esos años nace también en Pamplona el Instituto de Periodismo (1959), bajo el amparo del antiguo Estudio General de Navarra, y se funda la Escuela de Periodismo de la Iglesia (1960), auspiciada por el cardenal Ángel Herrera Oria, que ya había puesto en marcha en 1926 una academia orientada a la formación de periodistas vinculada al diario El Debate 2 .

Habrá que esperar hasta 1971 para que surjan las actuales facultades de Ciencias de la Información, en Madrid y Barcelona. Para los años 80, ya en democracia, se habían creado siete nuevos centros, a los que se sumaron doce más en la década de los 90. Finalmente, a partir del año 2000, los estudios de Periodismo se generalizan y dieciséis nuevas universidades los incorporan a su oferta académica. En la actualidad, se imparten en 37 campus españoles; de ellos, el 56,8\% son de titularidad pública y el $43,2 \%$, privada.

En general, la enseñanza del Periodismo se mueve entre distintos modelos, de desigual incidencia en el caso español ${ }^{3}$. El primero, que podemos denominar "tecnicista" (Cantarero, 2002: web), se centra en el desarrollo de habilidades profesionales por parte de los alumnos, con un diseño del currículo muy apegado a las demandas del mercado laboral. Es el tipo de estudios que se imparten de forma mayoritaria en los Estados Unidos y otros países de habla inglesa.

Frente a este, existe un modelo humanístico, "que enfatiza que el informador debe formarse con una solidez intelectual, proporcionada por las ciencias del hombre y de la sociedad" (Cantarero, 2002: web). En este caso, materias como la sociología, la filología, la semiótica o la teoría de la comunicación tienen mucho mayor peso en el programa de las titulaciones, y es la opción preferida por la mayoría de las universidades europeas.

Finalmente, hay una vía intermedia que busca reconciliar las aportaciones teóricas proporcionadas por las ciencias sociales y las humanidades con el cultivo de las destrezas técnicas requeridas en el ejercicio de la profesión.

En España, las primeras facultades, las fundadas a lo largo de la década de los setenta y principios de los ochenta, optaron mayoritariamente por el modelo humanístico, por una razón obvia: al no existir tradición académica en el área de las Ciencias de la Información, los responsables de las nuevas licenciaturas, procedentes en su totalidad de otras áreas de conocimiento, carecían de formación específica en comunicación, lo cual, como es lógico, dejó su impronta en los planes de estudios. Esto, sumado a otros condicionantes, como la secular resistencia de la Universidad española a incorporar a personas procedentes del mundo profesional y la ausencia de un corpus de doctrina preciso (Merayo, 2000: 2), propiciaron la configuración de unos títulos repletos de carga teórica, poco prácticos.

1 Sobre la labor de la Escuela Oficial de Periodismo, véase Humanes, 2002.

2 En la protohistoria de los estudios de Periodismo españoles debe citarse, como pionero, al profesor Fernando Araujo, quien en 1887 abrió en Salamanca un curso privado que no tuvo continuidad (López, 2009: 298).

3 Para disponer de una visión global del estado de la enseñanza del periodismo en el mundo, consúltese Fröhlich y Holtz-Balcha (2003). 
No obstante, a medida que el Periodismo adquiere peso académico, los nuevos centros que van surgiendo tratan de compatibilizar la formación humanística tradicional con el desarrollo de las aptitudes del oficio, de acuerdo con esa "tercera vía" que describíamos anteriormente, de modo que las facultades creadas entre finales de los ochenta y principios de los noventa ofrecen a sus alumnos un repertorio de materias mucho más acorde con las exigencias del mercado de trabajo (López, 2009: 305). Éste ha sido el modelo que, de manera paulatina, se ha ido generalizando en nuestro país, aunque siempre sometido a las críticas tanto del entorno académico como del profesional, cada uno -con excepciones-favorable a un determinado tipo de enseñanza ${ }^{4}$.

\subsection{El debate en torno a la orientación de los estudios}

En el ámbito académico, preocupa, por un lado, que se despojen de contenido teórico los títulos de Periodismo, convertidos casi en un taller de ejercicios: "El problema es que las Facultades de Comunicación están empezando a olvidar el sector de las humanidades y buscan la práctica sobre cosas muy efímeras, que son las del ejercicio profesional" (Moragas, en Fuentes, 2012: web). De acuerdo con esta postura, la carrera no debe limitarse a inculcar conocimientos técnicos, sino que es crucial que ilustre también "sobre los procesos de comunicación, sus repercusiones e implicaciones sociales y sobre los contextos sociales en los que está inserta" (Murciano, en Lamuedra, 2005: 209).

Por otro lado, se exige la adecuación de los programas a la nueva realidad del ecosistema comunicativo, transformado de raíz por la revolución digital (Galdón, 1997; Dader, 2007).

Pero el debate sobre el diseño de los programas y las habilidades que los estudiantes de periodismo necesitan, supera las fronteras nacionales, y durante el último decenio son numerosas las reflexiones en Europa y Norteamércia en este sentido (Deuze, 2001; MacDonald, 2006; Frith y Meech, 2007; Claussen, 2009); al fin y al cabo, "aunque los sistemas de medios y las culturas periodísticas pueden diferir ampliamente, los cambios y retos con los que se enfrenta la formación en Periodismo en todo el mundo son enormemente similares" (Deuze, 2006: 19). Las críticas sobre la distancia entre la formación académica que se imparte en las universidades y las destrezas técnicas exigidas por la profesión se han vertido universalmente (Bales, 1992; Reese y Cohen, 2000; El-Nawawy, 2007).

También hay planteamientos maximalistas que van más allá de la confección del currículo y cuestionan el condicionamiento de los estudios a los intereses de la industria mediática. Mensing (2010: 513) sostiene que mantener las prácticas educativas que refuerzan un statu quo no satisfactorio hace un flaco servicio a la credibilidad de la universidad, especialmente, porque al enfocar la formación al patrón profesional de la industria -en plena crisis del sector-se perpetúan patrones educativos que

${ }^{4}$ La propia transición de un tipo de planes de estudio a otro estuvo lastrada por numerosos condicionantes, como la masificación, la falta de recursos y el uso de métodos didácticos poco adecuados para la formación de los periodistas (Ortega y Humanes, 2000: 158). 
separan artificialmente la teoría de la práctica y se reduce la habilidad de los estudiantes para hacer frente a los rápidos cambios del entorno.

De lo que no se duda en la actualidad es de la importancia de la enseñanza universitaria del Periodismo para mantener la salud de la profesión y, sobre todo, de los profesionales, que de otro modo se ven abocados al anquilosamiento intelectual: "Las facultades de Ciencias de la Información y las Escuelas de Periodismo de Posgrado, si quieren ser efectivas -si combinan un equilibrio entre el conocimiento humanístico y la técnica periodística- han de ser sin duda cada vez más relevantes, ya que en las salas de redacción no se invierte lo suficiente en formación" (Sánchez, 2005: 86).

\subsection{La adaptación al EEES}

Los estudios universitarios en Europa están inmersos en un proceso de reforma que también afecta, como es lógico, al título de Periodismo, cuya circunscripción a la educación superior hace dos décadas que el Consejo de Europa sancionó ${ }^{5}$. El llamado Espacio Europeo de Educación Superior (EEES) representa un cambio de modelo radical, puesto que en los nuevos grados "la acumulación de conocimiento pierde relevancia (...) a favor de una cualificación más integral, entendida no sólo como un equilibrio entre el acopio de información o conocimiento y el dominio de destrezas profesionales. Se trata, en síntesis, de sintonizar el saber con el saber estar, el saber hacer y el saber ser" (Túñez et al., 2010: 87).

El nuevo sistema se implantó en 2010 en la totalidad de los estudios de Periodismo en España, así como en el resto de titulaciones de Ciencias de la Comunicación: Publicidad y Relaciones Públicas y Comunicación Audiovisual.

El objetivo final es favorecer la movilidad internacional de los profesionales de los 46 países adscritos al EEES, si bien existen problemas de convergencia que, prevemos, resultarán difíciles de soslayar, como apunta Hanitzsch, ya que "el ecosistema mediático influye en cada país en la visión y en la práctica del periodismo, dificultando los tránsitos hacia otras culturas y a los mercados laborales internacionales" (2008: $413)^{6}$.

El grado en Periodismo "no mantiene una referencia teórica sino que se basa en la búsqueda empírica de las competencias profesionales que deben poseer los egresados" (Pestano et al., 2011: 409). De acuerdo con el Libro Blanco de los Títulos de Grado de Comunicación (2005), los nuevos estudios pretenden dotar a los alumnos de

5 El Código Deontológico Europeo de la Profesión Periodística recoge, en su artículo 31, lo siguiente: "Dada la complejidad del proceso informativo, basado cada vez más en la utilización de nuevas tecnologías, la rapidez y la síntesis, se debe exigir a los periodistas una formación profesional adecuada" (en López, 2009: 296). Solo Gran Bretaña se mantiene al margen, con excepciones, de la enseñanza universitaria del Periodismo (véase Elías, 2009), prefiriendo la formación humanística o científica de base complementada con rudimentos de comunicación obtenidos a través de másteres.

6 El hecho de que el grado en España tenga una duración de cuatro años ya resulta anómalo con respecto a Europa, cuyos títulos se completan en tres. Se trata de un condicionante más que puede dificultar la tan ansiada convergencia internacional. 
capacidad analítico-crítica, buena preparación técnica y profesional, experimentación derivada de la práctica en laboratorios, reflexión sobre el quehacer periodístico, predisposición a la innovación y facilidad para adaptarse a los cambios. Asimismo, se contempla entre las exigencias la realización de prácticas en empresas y el dominio de lenguas extranjeras, la interdisciplinariedad, la capacidad de investigación y análisis y el dominio de las nuevas tecnologías.

En dos años se habrá completado la implantación del nuevo grado en todas las facultades españolas, y los esquemas tradicionales que han guiado la enseñanza del Periodismo en nuestro país habrán sufrido para entonces una completa transformación.

\subsection{Aproximaciones académicas a la opinión de los profesionales sobre los estu- dios de Periodismo}

La investigación sobre la profesión periodística solo empieza a acometerse en España a partir de la década de los noventa, y en principio, no atiende a la valoración que les merece a los redactores la formación universitaria recibida. El trabajo pionero en este campo, La élite de los periodistas, se limitaba a constatar que el $85 \%$ de los profesionales en ejercicio disponía de estudios superiores, aunque sin discriminar por áreas (Diezhandino et al., 1994: 55).

García de Cortázar y García de León (2000) ofrecen, a partir de 660 encuestas y una veintena de entrevistas en profundidad, datos más depurados: el 76,1\% de los trabajadores de los medios eran licenciados en ese momento, de los cuales un $69,2 \%$ disponían del título en Periodismo.

Ese mismo año, Canel et al. (2000: 18) ponen de manifiesto, en un trabajo apoyado también en un extenso análisis cuantitativo, que el 75,1\% de los periodistas españoles son licenciados en Ciencias de la Información, elevándose hasta el 92,1\% los que tienen algún tipo de formación en Comunicación. Se trata de un porcentaje inusitadamente alto en comparación con los que en 2000 presentaban, por ejemplo, Chile (70\%), Estados Unidos (39\%), Finlandia (25\%) o Gran Bretaña (4\%).

En este trabajo -y aquí radica para nosotros su principal interés- se pedía a los encuestados que valoraran la formación recibida en las facultades. En una escala de 1 ("irrelevante") a 6 ("importante"), más de la mitad, el 60,3\%, contestaron con valores 4, 5 y 6 (2000: 20). Canel et al. hacen notar que el escepticismo hacia la carrera ya recogido de forma asistemática en publicaciones previas- se va diluyendo con el paso del tiempo, merced, sobre todo, al incremento del contacto con el mundo empresarial y al papel de vanguardia que la universidad desempeña en relación con las nuevas tecnologías.

Finalmente, el Informe anual de la profesión periodística, que la Asociación de la Prensa de Madrid ha venido publicando de manera ininterrumpida desde 2004 hasta 2011, incluye una encuesta anual en la que se analizan, entre otros aspectos, las características sociodemográficas de los profesionales, su situación laboral, la evolución del entorno de trabajo y su grado de satisfacción con la formación recibida.

En general, la valoración de los estudios universitarios se ha mantenido estable a lo largo de los últimos ocho años [tabla 1], con el calificativo de "regular" como respuesta más frecuente, aunque los índices han experimentado una evolución ascen- 
dente en el caso de las opiniones favorables ("buena" y "muy buena"), que han ganado 10,8 puntos porcentuales, y decreciente para las opciones negativas ("mala" y "muy mala"), cuya pérdida ha sido de 18,4 puntos.

Tabla 1: Valoración de la enseñanza recibida en la Universidad.

Fuente: Informe anual de la profesión periodística (2004-2011)

\begin{tabular}{|l|c|c|c|c|c|c|c|c|}
\hline & $\mathbf{2 0 1 1}$ & $\mathbf{2 0 1 0}$ & $\mathbf{2 0 0 9}$ & $\mathbf{2 0 0 8}$ & $\mathbf{2 0 0 7}$ & $\mathbf{2 0 0 6}$ & $\mathbf{2 0 0 5}$ & $\mathbf{2 0 0 4}$ \\
\hline Muy buena & $4,50 \%$ & $0,70 \%$ & $3,00 \%$ & $2,90 \%$ & $4,60 \%$ & $1,80 \%$ & $3,00 \%$ & $0,80 \%$ \\
\hline Buena & $26,90 \%$ & $22,00 \%$ & $25,90 \%$ & $22,80 \%$ & $27,20 \%$ & $23,30 \%$ & $20,40 \%$ & $19,80 \%$ \\
\hline Regular & $41,10 \%$ & $45,40 \%$ & $41,20 \%$ & $45,50 \%$ & $42,40 \%$ & $43,40 \%$ & $40,40 \%$ & $39,20 \%$ \\
\hline Mala & $14,80 \%$ & $17,50 \%$ & $15,50 \%$ & $16,90 \%$ & $14,80 \%$ & $16,20 \%$ & $19,00 \%$ & $28,60 \%$ \\
\hline Muy mala & $4,70 \%$ & $5,60 \%$ & $6,60 \%$ & $6,80 \%$ & $4,70 \%$ & $7,80 \%$ & $8,70 \%$ & $9,30 \%$ \\
\hline Ns/nc & $8,20 \%$ & $8,80 \%$ & $7,80 \%$ & $5,10 \%$ & $6,30 \%$ & $7,50 \%$ & $8,50 \%$ & $2,40 \%$ \\
\hline
\end{tabular}

Fuera de España, algunos estudios recientes se han interesado por la opinión de los egresados en ejercicio sobre sus estudios, con disparidad de resultados: redactores y gerentes de los medios encuestados por Huang et al. (2006) coincidieron en valorar mejor las capacidades relacionadas con el pensamiento crítico que las técnicas. En Eslovenia, los periodistas entrevistados por Poler y Laban (2009) señalaron, en cambio, que la mayor deficiencia de su currículo universitario era la falta de conocimientos de tipo práctico. En Noruega, donde la licenciatura están claramente orientada al ejercicio profesional, el $65 \%$ de los egresados que respondieron tres años después de terminar la carrera a un cuestionario elaborado por Bjørnsen et al. (2007) estaban satisfechos con el programa que cursaron. Sin embargo, el contenido de la enseñanza recibida que consideraban más útil para su trabajo era la ética en lugar de los aspectos de carácter instrumental.

\section{Objetivos e hipótesis}

Partiendo de los datos presentados en el epígrafe 1.4, a los autores nos interesaba matizar la valoración que los estudios universitarios reciben por parte de los periodistas, atendiendo a determinadas variables que consideramos relevantes.

Dicha matización se hace necesaria por varias razones. La primera es que las cifras disponibles en el Informe anual de la profesión no discriminan entre los encuestados que han cursado Periodismo y los que poseen otros títulos en Ciencias de la Comunicación.

La segunda es que a lo largo de las tres últimas décadas, los estudios de Periodismo han estado sometidos a una continua puesta al día para tratar de responder a las exigencias del mercado, con lo que la enseñanza impartida en las facultades ha tenido por fuerza que ser diferente para los periodistas veteranos y para los de menor trayectoria. A ello hay que sumar el hecho de que la tercera generación de facultades, las que nacen entre finales de los ochenta y principios de los noventa, diseñan sus planes de estudios con el propósito expreso de combinar la formación teórica con el ejercicio de habilidades prácticas.

Teniendo en cuenta estas consideraciones, nos proponemos detectar si los cambios en la orientación de la licenciatura de Periodismo han tenido incidencia en la opinión 
que los egresados expresan sobre ella. Y en línea con este objetivo, planteamos tres hipótesis para su verificación o refutación:

- Hipótesis 1 (H1) - Si las modificaciones en el diseño de las enseñanzas han resultado eficaces en su empeño por responder a las necesidades profesionales, ha de existir una valoración más positiva por parte de los periodistas jóvenes, frente a quienes llevan dos o tres décadas ejerciendo el oficio y, por tanto, se formaron de acuerdo con los primeros planes, de orientación humanística.

- Hipótesis 2 (H2) - Deben existir diferencias en los aspectos negativos y positivos de la titulación que cada generación de periodistas destaca, como consecuencia de los cambios apuntados, de modo que los profesionales más veteranos serán más críticos con su formación debido a la ausencia de contenidos prácticos, con muy poco peso en los primeros programas.

- Hipótesis 3 (H3) - La valoración general sobre la formación universitaria puede variar en función del medio en el que los encuestados desempeñan su labor, siendo más favorable entre los que se dedican al periodismo escrito, que ha sido tradicionalmente el que más atención ha merecido en los planes de estudios de la licenciatura.

\section{Metodología}

\subsection{Población, muestra y recolección de los datos}

Se efectuó un análisis secundario de los datos recabados por Farias (2011) mediante una encuesta telefónica realizada a una muestra representativa $(n=1.000)$ de los profesionales asociados a la Federación de Asociaciones de Periodistas de España, cuya población de referencia ascendía a 15.000 periodistas. Los individuos fueron seleccionados de forma aleatoria simple a partir de una lista de miembros de la FAPE. Una empresa de encuestas realizó el trabajo de campo entre agosto y septiembre de 2011. La tasa de respuesta fue del 58,20\%. En cuanto a las características de los entrevistados, el 54,45\% eran hombres y el 45,55\%, mujeres. Su edad media era de 43,79 años $(\mathrm{DT}=11,68)$ y llevaban un promedio de 19,17 años $(\mathrm{DT}=11,41)$ ejerciendo la profesión. E1 75,32\% poseía un título universitario en Periodismo; el 15,88\% había estudiado otra especialidad de Ciencias de la Comunicación; el 5,59\% disponía de una licenciatura en un área de conocimiento diferente, y el 3,20\% restante carecía de estudios superiores.

Dado que el objeto de nuestro análisis solo atañe a quienes terminaron los estudios de Periodismo, se depuró la base de datos y se seleccionaron los casos que cumplían dicho requisito; de ahí que la muestra definitiva sea más reducida $(n=747)$. En este caso, el $50,20 \%$ de los individuos eran hombres y el $49,80 \%$, mujeres. Su edad media era de 42,72 años $(\mathrm{DT}=11,43)$ y acumulaban una media de 18,45 años de experiencia profesional $(\mathrm{DT}=11,17)$. Puesto que la licenciatura de Periodismo empezó a impartirse en España en el año 1971, pero no fue hasta 1976 cuando se graduó la primera promoción, los titulados más antiguos acumularían exactamente 35 años de experiencia profesional. De hecho, el $92,77 \%$ de los encuestados de la muestra definitiva llevaba 35 años o menos ejerciendo. El 7,23\% restante $(n=54)$ lo integraban periodistas en activo antes de 1976 y que, probablemente, decidieron ingresar en la Universidad con posterioridad a su incorporación al mercado de trabajo. 


\subsection{Variables del estudio}

a) Variables dependientes.

- Valoración de la formación recibida. Se empleó una variable ordinal de cinco puntos (5, muy buena; 1 , muy mala) para medir la opinión sobre la calidad de los estudios.

- Aspecto positivo destacado de la formación. Se formuló una pregunta abierta para recoger un aspecto que los periodistas destacaran como positivo de la licenciatura que cursaron. Las respuestas fueron categorizadas posteriormente; por tanto, se analizó como variable nominal.

- Aspecto negativo destacado de la formación. Idéntico procedimiento que en el caso anterior (pregunta abierta, categorización de las respuestas).

b) Variables independientes.

- Generación-4. Se crearon cuatro grupos entre los periodistas a partir de la variable "Experiencia profesional", recogida en años. Estimamos que los entrevistados con una experiencia de entre 0 y 11 años estudiaron durante la década de 2000 (2011-2001) (1= Grupo 2000); los periodistas con una experiencia de entre 12 y 21 años, durante la década de los 90 (2000-1991) (2=Grupo 90); los encuestados con una experiencia de entre 22 y 31 años, durante la década de los 80 (1990-1981) (3= Grupo 80), y los periodistas con una experiencia superior a 32 años, en la década de los 70 (1980-1971) (4= Grupo 70).

- Generación-2. Se creó también una versión reducida de la variable Generación4 con la que se agrupó a los periodistas en dos conjuntos. Estimamos que los encuestados con una trayectoria igual o inferior a 21 años estudiaron entre 1990 y 2011, y por tanto, se formaron con los planes de estudios reformados $(1=$ Grupo más joven); y que aquellos con una experiencia igual o superior a 22 años cursaron la carrera con anterioridad a la última década del siglo XX, sometiéndose a los programas de corte humanístico ( $2=$ Grupo más veterano).

Obviamente, estas dos variables tienen limitaciones. En el periodismo era habitual, hasta que las facultades se generalizaron hace dos décadas, que algunos profesionales comenzaran a trabajar antes de incorporarse a la Universidad, o que empezaran a ejercer después de abandonar la carrera durante un tiempo. No obstante, al carecer de datos que indiquen la fecha de obtención del título, las variables utilizadas en esta investigación nos parecen las más razonables con los datos disponibles.

- Tipo de medio en el que trabaja. Se trata de una variable categórica que identifica el tipo de medio donde el periodista ejerce principalmente su profesión $(1=$ periódicos; $2=$ revistas; $3=$ radio; $4=$ televisión; $5=$ ediciones digitales; $6=$ agencias de noticias; $7=$ departamentos de comunicación y gabinetes de prensa).

\subsection{Procedimiento de análisis}

Tras seleccionar los casos elegibles y fijar la muestra definitiva $(n=747)$, se utilizó el método ANOVA de un factor con un nivel de significación alfa para comparar las medias de la variable "Valoración de la formación recibida" entre los grupos determinados como "Generación-4". Se recurrió a este mismo procedimiento para cotejar la opinión expresada por los periodistas según "Tipo de medio en el que trabaja". 
Se emplearon tablas de contingencia con porcentajes de columna para cruzar las variables "Aspecto positivo destacado de la formación" y "Aspecto negativo destacado de la formación" entre los grupos de "Generación-2". Finalmente, se realizó la prueba $\mathrm{Z}$ con la corrección de Bonferroni para comparar las proporciones de columna.

\section{Resultados}

\subsection{Valoración de la formación recibida según la generación del periodista}

La mayoría de los periodistas de la muestra $(44,31 \%)$ calificaron la enseñanza recibida en la Universidad como "regular"; el 22,09\% la consideró "mala" o "muy mala"; y el 33,60\%, "buena" o "muy buena".

La H1 sugería que habría diferencias significativas en la valoración expresada por las distintas generaciones de profesionales encuestados. Asimismo, se esperaba que los periodistas jóvenes mantuvieran una opinión más favorable hacia la titulación que los veteranos.

La prueba ANOVA de un factor indicó que no había diferencias significativas entre los grupos determinados por la variable "Generación-4" en las puntuaciones medias de "Valoración de la formación recibida" a un nivel de significación del 0,05 $[F(3,743)=2,58 ; p=0.052]$ [Tabla 2]. Por tanto, se sugiere rechazar H1.

Tabla 2: Valoración de la formación recibida

\begin{tabular}{|c|c|c|c|c|c|c|c|c|}
\hline \multicolumn{9}{|c|}{ Medias según su grupo en Generación-4 } \\
\hline \multirow{2}{*}{ Año } & \multirow{2}{*}{$\mathbf{N}$} & \multirow{2}{*}{ Media } & \multirow{2}{*}{$\begin{array}{l}\text { Desviación } \\
\text { estándar }\end{array}$} & \multirow{2}{*}{$\begin{array}{c}\text { Error } \\
\text { estándar }\end{array}$} & \multicolumn{2}{|c|}{$\begin{array}{c}\text { Intervalo de confianza para la } \\
\text { media del } 95 \%\end{array}$} & \multirow{2}{*}{ Mín. } & \multirow{2}{*}{ Máx. } \\
\hline & & & & & Cota inferior & Cota superior & & \\
\hline 2000 & 221 & 3,21 & 0,927 & 0,062 & 3,09 & 3,34 & 1 & 5 \\
\hline 1990 & 222 & 2,99 & 0,939 & 0,063 & 2,86 & 3,11 & 1 & 5 \\
\hline 1980 & 203 & 3,07 & 0,890 & 0,062 & 2,95 & 3,20 & 1 & 5 \\
\hline 1970 & 101 & 3,19 & 0,924 & 0,092 & 3,01 & 3,37 & 1 & 5 \\
\hline Total & 747 & 3,10 & $\mathbf{0 , 9 2 3}$ & $\overline{0,034}$ & 3,04 & 3,17 & 1 & 5 \\
\hline
\end{tabular}

4.2. Opinión sobre aspectos positivos y negativos según la generación del periodista

Tabla 3: Aspecto positivo destacado de la formación recibida * Generación-2

\begin{tabular}{|c|c|c|c|c|}
\hline \multicolumn{5}{|c|}{$\%$ de media según su grupo en Generación-2 } \\
\hline \multirow{9}{*}{$\begin{array}{l}\text { Aspecto } \\
\text { positivo } \\
\text { destacado de la } \\
\text { formación } \\
\text { recibida }\end{array}$} & \multirow[b]{2}{*}{ Criterios } & \multicolumn{2}{|c|}{ Generación-2 } & \multirow[b]{2}{*}{ Total } \\
\hline & & $\begin{array}{c}\text { Grupo más } \\
\text { joven }\end{array}$ & $\begin{array}{c}\text { Grupo más } \\
\text { veterano }\end{array}$ & \\
\hline & Cultura/Conocimientos & $27,59 \% \%_{a}$ & $39,06 \% \%_{\mathrm{b}}$ & $32,02 \%$ \\
\hline & Prácticas & $20,94 \% \%_{a}$ & $8,20 \%{ }_{b}$ & $16,01 \%$ \\
\hline & Profesores & $12,81 \% \%_{a}$ & $13,28 \% \%_{a}$ & $12,99 \%$ \\
\hline & Multidisciplinariedad & $9,36 \% \%_{a}$ & $10,94 \% \%_{a}$ & $9,97 \%$ \\
\hline & Ninguno & $7,14 \% \%_{a}$ & $7,42 \% \mathrm{a}_{\mathrm{a}}$ & $7,25 \%$ \\
\hline & Contacto con la profesión & $5,67 \% \%_{a}$ & $3,91 \% \%_{a}$ & $4,98 \%$ \\
\hline & Compañeros/Ambiente & $3,45 \%$ & $5,08 \%$ a & $4,08 \%$ \\
\hline
\end{tabular}




\begin{tabular}{|c|c|c|c|c|}
\hline & Ética/Valores & $3,45 \%_{\mathrm{a}}$ & $3,13 \% \%_{a}$ & $3,32 \%$ \\
\hline & Contacto con los medios & $3,20 \% \%_{a}$ & $1,17 \%_{a}$ & $2,42 \%$ \\
\hline & Otros & $6,40 \% \%_{a}$ & $7,81 \% \%_{a}$ & $6,95 \%$ \\
\hline & Total & $100 \%$ & $100 \%$ & $100 \%$ \\
\hline
\end{tabular}

Tabla 4:Aspecto negativo destacado de la formación recibida * Generación-2

\begin{tabular}{|c|c|c|c|c|}
\hline \multicolumn{5}{|c|}{ \% de media según su grupo en Generación-2 } \\
\hline \multirow{12}{*}{$\begin{array}{l}\text { Aspecto } \\
\text { negativo } \\
\text { destacado de la } \\
\text { formación } \\
\text { recibida }\end{array}$} & \multirow[b]{2}{*}{ Criterios } & \multicolumn{2}{|c|}{ Generación-2 } & \multirow[b]{2}{*}{ Total } \\
\hline & & $\begin{array}{c}\text { Grupo más } \\
\text { joven }\end{array}$ & $\begin{array}{c}\text { Grupo más } \\
\text { veterano }\end{array}$ & \\
\hline & Falta de contenidos prácticos & $30,58 \% \%_{a}$ & $38,52 \% \mathrm{o}_{\mathrm{b}}$ & $33,63 \%$ \\
\hline & Alejada de la realidad & $17,72 \%_{\mathrm{a}}$ & $12,84 \%_{\mathrm{a}}$ & $15,84 \%$ \\
\hline & Profesorado & $7,28 \% \%_{a}$ & $10,89 \% \%_{a}$ & $8,67 \%$ \\
\hline & Falta de especialización & $8,98 \% \mathrm{o}_{\mathrm{a}}$ & $6,61 \% \%_{a}$ & $8,07 \%$ \\
\hline & Falta de medios & $6,07 \%_{\mathrm{a}}$ & $7,39 \% \%_{a}$ & $6,58 \%$ \\
\hline & Masificación & $6,31 \%_{a}$ & $5,06 \%{ }_{a}$ & $5,83 \%$ \\
\hline & Demasiada teoría & $4,61 \%{ }_{a}$ & $4,67 \% \%_{a}$ & $4,63 \%$ \\
\hline & Falta de idiomas & $3,64 \% \mathrm{o}_{\mathrm{a}}$ & $3,50 \% \mathrm{a}_{\mathrm{a}}$ & $3,59 \%$ \\
\hline & Asignaturas inútiles & $4,13 \% \mathrm{a}_{\mathrm{a}}$ & $1,95 \%{ }_{a}$ & $3,29 \%$ \\
\hline & Mala enseñanza/Incompleta & $3,64 \% \%_{a}$ & $0,78 \%$ b & $2,54 \%$ \\
\hline & Total & $100 \%$ & $100 \%$ & $100 \%$ \\
\hline
\end{tabular}

El aspecto positivo destacado por un porcentaje mayor de periodistas fue "La cultura/Los conocimientos" adquiridos (32\%), seguido de "Las prácticas" (16\%) y "Los profesores" (13\%). El resto de características fueron mencionadas por menos de un $10 \%$ de encuestados [Tabla 3]. Por otra parte, la cualidad negativa citada por un mayor número de profesionales fue "La falta de contenidos prácticos" (33\%), seguida de "Alejada de la realidad" $(15,8 \%)$. Ninguna otra propiedad fue destacada por más de un $10 \%$ de entrevistados [Tabla 4 ].

La H2 establecía que habría diferencias en la opinión sobre los aspectos positivos y negativos de la formación recibida entre el grupo de periodistas que estudiaron antes de los 90 y quienes se formaron en las décadas posteriores.

Tal y como se recoge en la Tabla 3, la prueba $\mathrm{Z}$ indicó la existencia de divergencias apreciables en los porcentajes referidos a "Aspecto positivo de la formación" entre los dos grupos determinados por la variable "Generación-2". Los resultados demuestran que la tasa de periodistas del colectivo más veterano que señaló como elemento positivo "La cultura/Los conocimientos" fue notablemente mayor $(39,06 \%)$ que la del grupo más joven $(27,59 \%)$. A la inversa, el número de profesionales con menor trayectoria que mencionó como aspecto positivo "Las prácticas" fue significativamente más alto $(20,94 \%)$ que el del grupo que llevaba al menos 22 años ejerciendo la profesión $(8,20 \%)$. 
Por otra parte, de acuerdo con la información proporcionada en la Tabla 4, la prueba $\mathrm{Z}$ demuestra de nuevo diferencias de calado en los porcentajes correspondientes al "Aspecto negativo de la formación" entre los grupos de la variable "Generación-2". Así, el índice de periodistas veteranos que se quejó de "La falta de contenidos prácticos" era notablemente mayor (38,52\%) que el de los periodistas que estudiaron en las dos últimas décadas (30,58\%). Todas las evidencias mencionadas soportan la $\mathrm{H} 2$, ya que los encuestados que pasaron por la Facultad cuando aún estaban vigentes los primeros planes de estudios valoró más la formación humanística recibida que los jóvenes y criticó en mayor proporción el déficit de asignaturas prácticas.

\subsection{Valoración de la formación recibida según el tipo de medio en el que trabaja} La H3 establecía que habría diferencias claras en la valoración expresada por los periodistas dependiendo de que desempeñaran su labor en diarios, revistas, radios, televisiones, agencias, medios digitales o gabinetes de comunicación. Así, se entendía que los profesionales vinculados a la prensa escrita tendrían mejor opinión de la enseñanza universitaria que el resto.

Los resultados de la prueba ANOVA de un factor revelan que no hay divergencias significativas en las puntuaciones medias obtenidas en esta pregunta entre los grupos de periodistas determinados por "Tipo de medio en el que trabaja" $[\mathrm{F}(6,728)=0,47$; $\mathrm{p}=0.829]$. En consecuencia, se refuta $\mathrm{H} 3$.

\section{Conclusiones y discusión}

La licenciatura de Periodismo en España ha estado sometida a un continuo proceso de reformulación, con el propósito de adaptarse a las necesidades de sus egresados de cara al ejercicio profesional. De este modo, desde 1971 han funcionado diferentes planes de estudios, unos -los primeros- más volcados con la formación humanística de los futuros informadores, y otros -implantados hace en torno a dos décadas- más proclives al cultivo de las habilidades técnicas y las aptitudes que exige el trabajo diario en los medios.

Nuestro propósito en esta ponencia estribaba en determinar eventuales divergencias entre la percepción que los periodistas tienen sobre su formación universitaria dependiendo de la generación a la que pertenecen y el medio al que están adscritos en la actualidad. El análisis de los datos recabados nos ha dado la razón sólo en parte.

1. Con respecto a la primera hipótesis, referida a la valoración general que se otorgaba a la licenciatura, hemos constatado que no hay diferencias entre los que estudiaron en la década de 2000 (2011-2001), en los 90 (2000-1991), en los 80 (1990-1981) y en los 70 (1980-1971). Y tampoco apreciamos disimilitudes una vez agrupados los periodistas según cursaran la carrera con los primeros planes de estudios o con los programas reformados de finales de los 80 y principios de los 90 . Para descartar que las coincidencias se debieran a la forma en que se habían creado los grupos, también se comparó la valoración según los tramos de edad de los periodistas, y el resultado fue igualmente negativo.

Dado que la opinión mayoritaria sobre la formación en todas las generaciones es "regular", con un alto nivel de acuerdo entre los encuestados, podemos pensar que 
los cambios aplicados a los estudios de Periodismo a lo largo de sus 40 años de historia han conseguido mantener unos estándares de satisfacción entre los licenciados suficientes aunque claramente mejorables. No obstante, la ausencia de divergencias claras entre unos grupos de entrevistados y otros también pone de manifiesto la incapacidad del sistema universitario para conseguir una mejora sustancial en la percepción de sus egresados sobre la calidad de la enseñanza recibida. Quienes finalizaron su carrera en 2011 no consideran haber recibido peor ni mejor formación que aquellos que terminaron en 1976: es una constatación que invita a reflexionar sobre la eficacia de las remodelaciones curriculares acometidas en estos años.

2. Sí que se han apreciado diferencias generacionales en relación con los aspectos positivos y negativos que cada grupo destaca. Así, los periodistas de las décadas de los 70 y 80 valoran más "La cultura y los conocimientos teóricos" adquiridos, mientras que los profesionales jóvenes se muestran satisfechos en mayor medida con el componente práctico de la carrera.

Asimismo, el grupo de encuestados más veterano achaca a sus estudios sobre todo la falta de materias de índole técnica, en tanto que para los periodistas de las décadas de los 90 y 2000 una disfunción importante de la licenciatura es que no responde a la realidad de la profesión.

Aun así, todos los entrevistados coinciden en señalar que la ausencia o insuficiencia de prácticas es el principal inconveniente de la titulación.

3. No hay, finalmente, diferencias en la percepción de la licenciatura según se trabaje en un medio impreso, audiovisual o digital. Los conocimientos adquiridos y las carencias parecen ser cuantitativamente idénticas, lo cual no deja de ser llamativo si tenemos en cuenta que el aprendizaje del periodismo escrito dispone de un espacio generoso en todos los planes de estudios, mientras que la producción digital se ejercita en mucha menor medida, del mismo modo que la realización audiovisual, muy costosa en términos técnicos. Y aun así, todos los licenciados consideran que han accedido al mercado de trabajo con idéntico bagaje.

En definitiva, los estudios de Periodismo, cuatro décadas después de implantarse en España, siguen buscando el equilibrio entre contenidos teóricos y desarrollos prácticos sin solucionar muchos de los retos a los que los egresados se enfrentan una vez que comienzan a ejercer. El propósito de los nuevos grados inspirados por la Declaración de Bolonia se orienta a reducir la brecha entre el ámbito académico y el profesional, pero aún habrá que esperar algunos años para conocer si este empeño se ve coronado por el éxito.

\section{Referencias bibliográficas}

BALES, Fred (1992): "Newspaper editors' evaluations of professional programs". Journalism Educator, $\mathrm{n}^{\circ}$ 47. Pittsburgh, American Society of Journalism School Administrators, pp. 37-42.

BJØRNSEN, Gunn; HOVDEN, Jan Fredrik y OTTOSEN, Rune (2007): “Journalists in the making". Journalism Practice, n 1, 3. Cardiff, Routledge, pp. 383-403. 
CANEL, María José; SÁNCHEZ ARANDA, José Javier; y RODRÍGUEZ, Roberto (2000): Periodistas al descubierto. Retrato de los profesionales de la información. Madrid, Centro de Investigaciones Sociológicas.

CANTARERO, Mario Alfredo (2002): "Formación de comunicadores sociales. Modelos curriculares, ostracismo académico, rutas sociales y esperanzas". Revista Latina de Comunicación Social, $\mathrm{n}^{\mathrm{o}}$ 52: http://www.ull.es/publicaciones/latina /20025209CANTAREROxi.htm [fecha de consulta: 13 de febrero de 2012].

CLAUSSEN, Dane (2009): "How one would really 'blow up' a J-school curriculum". Journalism and Mass Communication Educator, $\mathrm{n}^{\circ}$ 64. Columbia, AEJMC, pp. 133-136.

CONFERENCIA DE DECANOS DE CIENCIAS DE LA INFORMACIÓN (2005): Libro Blanco de los Títulos de Grado de Comunicación: http://www.aneca.es /var/media/150336/libroblanco_comunicacion_def.pdf [fecha de consulta: $11 \mathrm{de}$ marzo de 2012].

DADER, José Luis (2006): "Reflexividad e innovación metodológicas como condición de progreso para las Ciencias de la Comunicación", en LOSADA, Ángel; PLAZA, Juan Francisco y HUERTA, Miguel Ángel (coords.): Comunicación, Universidad y Sociedad del Conocimiento. Salamanca, Universidad Pontificia de Salamanca.

DEUZE, Mark (2001): "Educating 'new' journalists: Challenges to the curriculum". Journalism and Mass Communication Educator, $\mathrm{n}^{\circ}$ 56. Columbia, AEJMC, pp. 417.

DEUZE, Mark (2006): “Global Journalism Education”. Journalism Studies, nº 7, 1. Cardiff, Routledge, pp. 19-34.

DIEZHANDINO, Pilar; BEZUNARTEA, Ofa; y COCA, César (1994): La élite de los periodistas. Bilbao, Ediciones de la Universidad del País Vasco.

EL-NAWAWY, Mohammed (2007): "Between the newsroom and the classroom: Education standards and practices for print journalism in Egyp and Jordan". The International Communication Gazette, $\mathrm{n}^{\mathrm{o}}$ 69. Londres, Thousand Oaks y Nueva Delhi, Sage, pp. 69-90.

ELÍAS, Carlos (2009): “¿Perjudica la carrera de Periodismo el buen quehacer informativo?", en DE PABLOS, José Manuel (ed.): Actas del I Congreso Internacional Latina de Comunicación Social. La Laguna, Revista Latina: http://www.revistalatinacs.org/09/Sociedad/actas/13elias.pdf [fecha de consulta: 27 de febrero de 2012].

FARIAS BATLLE, Pedro (dir., 2011): Informe anual de la profesión periodística. Madrid, Asociación de la Prensa de Madrid.

FUENTES, Raúl (2012): "La formación universitaria de profesionales de la comunicación y su renovación como proyecto social". Diálogos de la Comunicación, $\mathrm{n}^{\circ}$ 59-60: http://www.dialogosfelafacs.net/wp-content/uploads/2012/01/59-60-revistadialogos-la-formacion-universitaria.pdf [fecha de consulta: 2 de febrero de 2012]. 
FRITH, Simon y MEECH, Peter (2007): "Becoming a journalist: Journalism education and journalism culture". Journalism, $\mathrm{n}^{\circ} 8$. London, Thousand Oaks y Nueva Delhi, Sage, pp. 137-164.

FRÖHLICH, Romy y HOLTZ-BACHA, Christina (2003): Journalism education in Europe and North America. An international comparison. New Jersey, Hampton Press.

GALDÓN, Gabriel (1997): La enseñanza del periodismo. Una propuesta de futuro. Barcelona, CIMS.

GARCÍA DE CORTÁZAR, Marisa y GARCÍA DE LEÓN, María Antonia (coords., 2000): Profesionales del periodismo. Hombres y mujeres en los medios de comunicación. Madrid, Centro de Investigaciones Sociológicas.

HANITZSCH, Thomas (2009): "Comparative Journalism Studies", en WAHL-JORGENSEN, Karin y HANITZSCH, Thomas (eds.): The Handbook of Journalism Studies. New York, Taylor \& Francis, pp. 413-427.

HUANG, Edgar; DAVIDSON, Karen; SHREVE, Stephanie; DAVIS, Twila; BETTENDORF, Elizabeth y NAIR, Anita (2006): "Facing the challenges of convergence. Media professionals' concerns of working across media platforms". Convergence Review, vol. 12 (1). Canberra, DBCDE, pp. 83-98.

HUMANES, María Luisa (2002): "La política de formación de comunicadores: La Escuela Oficial de Periodismo (1941-1970)", en GARCÍA GALINDO, Juan Antonio; GUTIÉRREZ LOZANO, Juan Francisco; y SÁNCHEZ ALARCÓN, Inmaculada (eds.): La comunicación social durante el franquismo. Málaga, CEDMA, pp. 625-642.

LAMUEDRA, María (2007): "Estudiantes de Periodismo y prácticas profesionales: el reto del aprendizaje". Comunicar, n 28. Huelva, Grupo Comunicar, pp. 203-2011.

LÓPEZ GARCÍA, Xosé (2009): "Tendencias en la formación de los periodistas en los ámbitos hispanos y lusófonos en el siglo XXI". Estudios sobre el mensaje periodístico, vol. 15. Madrid, Servicio de Publicaciones de la Universidad Complutense, pp. 295-313.

LÓPEZ GARCÍA, Xosé (2010): "La formación de los periodistas en el siglo XXI en Brasil, España, Portugal y Puerto Rico". Revista Latina de Comunicación Social, $\mathrm{n}^{\mathrm{o}}$ 65, pp. 231-243: http://www.revistalatinacs.org/10/art2/896_Santiago/18 _Xose.html. [fecha de consulta: 14 de febrero de 2012].

MACDONALD, Isabel (2006): "Teaching journalists to save the profession: a critical assessment of recent debates on the future of US and Canadian journalism education". Journalism Studies, n 7 . Cardiff, Routledge, pp. 745-764.

MENSING, Donica (2010): "Rethinking (again) the future of Journalism Education". Journalism Studies, ${ }^{\circ}$ 11, 4. Cardiff, Routledge, pp. 511-523.

MERAYO, Arturo (2000): "Periodistas para el siglo de la información: Claves para formar a los nuevos comunicadores". BOCC, Biblioteca Online de Ciências da Co- 
municação: http://www.bocc.ubi.pt/pag/merayo-arturo-formacion-periodistasXXI.pdf [fecha de consulta: 5 de marzo de 2012].

ORTEGA, Félix y HUMANES, María Luisa (2000): "Periodistas del siglo XXI. Sus motivaciones y expectativas profesionales". Cuadernos de Información y Comunicación, $\mathrm{n}^{\mathrm{o}}$ 5. Madrid, Servicio de Publicaciones de la Universidad Complutense, pp. 153-170.

PESTANO, José Manuel; RODRÍGUEZ, Carmen; y DEL PONTI, Patricia (2011): "Transformaciones en los modelos de formación de periodistas en España. El reto europeo". Estudios sobre el mensaje periodístico, vol. 17, núm. 2. Madrid, Servicio de Publicaciones de la Universidad Complutense, pp. 401-415.

POLER, Melita y LABAN, Vesna (2009): “Journalism Education in Slovenia”. Journalism Practice, $\mathrm{n}^{\mathrm{o}}$ 3, 1. Cardiff, Routledge, pp. 92-107.

REESE, Stephen y COHEN, Jeremy (2000): "Education for Journalism: the professionalism of scholarship". Journalism Studies, n ${ }^{\circ}$ 1, 2. Cardiff, Routledge, pp. 213227.

SÁNCHEZ ILLÁN, Juan Carlos (2005): "El perfil profesional del periodista español. Evolución reciente y nuevos desafíos (1990-2005)". Telos, $\mathrm{n}^{\circ}$ 63. Madrid, Fundación Telefónica, pp. 85-92.

TÚÑEZ, Miguel; MARTÍNEZ, Yolanda; y ABEJÓN, Paloma (2010): "Nuevos entornos, nuevas demandas, nuevos periodistas". Estudios sobre el mensaje periodístico, vol. 16. Madrid, Servicio de Publicaciones de la Universidad Complutense, pp. 79-94. 